\title{
Implications of human evolution and admixture for mitochondrial replacement therapy
}

\author{
Lavanya Rishishwar ${ }^{1,2,3}$ and I. King Jordan ${ }^{1,2,3^{*}}$ (D)
}

\begin{abstract}
Background: Mitochondrial replacement (MR) therapy is a new assisted reproductive technology that allows women with mitochondrial disorders to give birth to healthy children by combining their nuclei with mitochondria from unaffected egg donors. Evolutionary biologists have raised concerns about the safety of MR therapy based on the extent to which nuclear and mitochondrial genomes are observed to co-evolve within natural populations, i.e. the nuclear-mitochondrial mismatch hypothesis. In support of this hypothesis, a number of previous studies on model organisms have provided evidence for incompatibility between nuclear and mitochondrial genomes from divergent populations of the same species.
\end{abstract}

Results: We tested the nuclear-mitochondrial mismatch hypothesis for humans by observing the extent of naturally occurring nuclear-mitochondrial mismatch seen for 2,504 individuals across 26 populations, from 5 continental populations groups, characterized as part of the 1000 Genomes Project (1KGP). We also performed a replication analysis on mitochondrial DNA (mtDNA) haplotypes for 1,043 individuals from 58 populations, characterized as part of the Human Genome Diversity Project (HGDP). Nuclear DNA (nDNA) and mtDNA sequences from the 1KGP were directly compared within and between populations, and the population distributions of mtDNA haplotypes derived from both sequence (1KGP) and genotype (HGDP) data were evaluated. Levels of nDNA and mtDNA pairwise sequence divergence are highly correlated, consistent with their co-evolution among human populations. However, there are numerous cases of co-occurrence of nuclear and mitochondrial genomes from divergent populations within individual humans. Furthermore, pairs of individuals with closely related nuclear genomes can have highly divergent mtDNA haplotypes. Supposedly mismatched nuclear-mitochondrial genome combinations are found not only within individuals from populations known to be admixed, where they may be expected, but also from populations with low overall levels of observed admixture.

Conclusions: These results show that mitochondrial and nuclear genomes from divergent human populations can co-exist within healthy individuals, indicating that mismatched nDNA-mtDNA combinations are not deleterious or subject to purifying selection. Accordingly, human nuclear-mitochondrial mismatches are not likely to jeopardize the safety of MR therapy.

Keywords: mtDNA, Population genomics, Three-person baby

\footnotetext{
* Correspondence: king.jordan@biology.gatech.edu

${ }^{1}$ School of Biology, Georgia Institute of Technology, Atlanta, GA, USA

${ }^{2}$ PanAmerican Bioinformatics Institute, Cali, Colombia

Full list of author information is available at the end of the article
}

(c) The Author(s). 2017 Open Access This article is distributed under the terms of the Creative Commons Attribution 4.0 International License (http://creativecommons.org/licenses/by/4.0/, which permits unrestricted use, distribution, and reproduction in any medium, provided you give appropriate credit to the original author(s) and the source, provide a link to the Creative Commons license, and indicate if changes were made. The Creative Commons Public Domain Dedication waiver (http://creativecommons.org/publicdomain/zero/1.0/) applies to the data made available in this article, unless otherwise stated. 


\section{Background}

Mutations to mitochondrial DNA (mtDNA) have been associated with a wide range of human diseases $[1,2]$. Since mitochondria are maternally inherited, mitochondrial genetic disorders will be passed from mothers to their children. Effective treatments for mitochondrial disease are rare, and patients are often faced with limited therapeutic options. Furthermore, the ability to accurately assess the risk of inheriting a mitochondrial genetic disorder can be complicated by the co-occurrence of wild-type and mutated mtDNA (i.e., heteroplasmy) in a single female [3]. Mitochondrial replacement (MR) therapy is a promising new assisted reproductive technology that could allow women with mitochondrial disorders to give birth to healthy children to whom they are closely genetically related. MR therapy works by combining nuclear DNA (nDNA) from a mother who has a mitochondrial disorder together with healthy mitochondria from an egg donor. For MR-assisted in vitro fertilization (IVF), the nuclear genome is removed from a fertilized oocyte with diseased mitochondria and injected into an enucleated donor egg that contains healthy mitochondria. This process results in so-called 'three-person babies' since children born from MR therapy will have genetic contributions from two mothers and one father.

Studies in mammalian systems over the last decade have underscored both the surmountable technical challenges, and the considerable promise, associated with MR therapy. The nuclear transplantation procedure that underlies MR therapy was first shown to be possible in mice [4]. The progeny of nuclear transplantations from mouse oocytes with mitochondrial disease into healthy oocytes were found to be viable and disease-free. Later in primates, MR therapy was used to produce four Macaque offspring [5] that showed healthy development to 3 years of age [6]. Nuclear transfer in this case occurred prior to fertilization, a technique that has not proven to be equally effective in humans. The MR procedure was first developed in humans using abnormally fertilized zygotes [7]. Human MR experiments rely on pronuclear transfer, whereby the nuclear genome is removed from a newly formed human embryo shortly after fertilization. This approach showed promise with respect to both the small amount of diseased mitochondria that are carried over to the healthy donor egg and in terms of normal in vitro development through the blastocyst stage. More recently, the same group demonstrated even greater efficacy of the pronuclear transfer technique for MR therapy with normally fertilized embryos by transferring the pronuclei compartments containing maternal and paternal haploid genomes almost immediately after they first appear [8].

These crucial experimental advances in MR therapy have occurred against a backdrop of substantial regulatory investigation related to the technique's desirability, safety and potential efficacy [9]. Most of the effort and progress on this front has occurred in the United Kingdom (UK). The UK's Human Fertilisation and Embryology Authority (HFEA) was initially charged with evaluating MR therapy, and they recommended further studies before the technique could be adopted as a clinical practice. Subsequently, several independent UK science agencies supported the bioethics of MR therapy, and the public was found to be largely in favor of its use. These findings ultimately led the UK government to draft a set of regulations for the technique, and parliament approved MR therapy as an assisted reproductive technology in February of 2015. Regulations were to be enacted by October of the same year, with clinics able to apply for a license by November. Initial attempts to conceive via MR-assisted IVF could have begun by the end of that same year. When this manuscript was written, there was no record of any human birth resulting from MR therapy in the UK. However, during the manuscript review process, news broke of a 'three-person baby' resulting from MR therapy born in Mexico [10]. The United States (US) doctors who led the procedure chose Mexico to avoid US regulations that do not yet permit MR therapy, leading to charges of unethical and irresponsible behavior.

Despite the considerable technical and regulatory progress that has been made on the issue, substantial concerns have been raised about the safety of MR therapy $[9,11]$. These concerns rest largely on the notion of potential incompatibility (mismatches) between nuclear and mitochondrial genomes from different populations of the same species. We refer to this idea here as the 'nuclearmitochondrial mismatch hypothesis'. Incompatibility between nuclear and mitochondrial genomes from divergent populations would most likely be predicated upon interactions between proteins encoded by each [11]. While the human mitochondrial genome only encodes 37 protein coding genes, there are more than a thousand nuclear genes that encode proteins involved in mitochondrial function. These include 76 nuclear encoded proteins that directly bind mitochondrial counterparts. Sequence variations that change the binding affinities between nuclear and mitochondrial proteins could have deleterious effects, which would jeopardize healthy outcomes from MR therapy. This possibility has been supported by comparative sequence analyses showing the importance of compensatory sequence changes that serve to maintain physical interactions between nuclear and mitochondrial encoded proteins [12, 13].

A number of studies from model organisms have provided even more direct evidence for incompatibility between nuclear and mitochondrial genomes brought together from different populations of the same species. For example, mice with mismatched mitochondrial and 
nuclear genomes were able to survive to adulthood but showed stunted growth and reduced physical performance [14] as well as reduced learning and exploratory behavior [15]. The neurological effects observed in the latter study increased with age. Analogous studies in invertebrates have also turned up numerous deleterious effects of combining divergent nuclear and mitochondrial genomes. Such effects include changes in aging [16-19], survival [20] and fertility [21-24] along with impaired mitochondrial function $[25,26]$. It should be noted that all of these studies entailed repeated genetic backcrosses whereby divergent mitochondria were introduced into highly inbred lines. As such, they represent extremes of genetic divergence between lineages and are not likely to accurately reflect human populations that routinely interbreed [27]. Nevertheless, these findings do point to a number of possible complications arising from nuclearmitochondrial genome mismatch.

While the aforementioned studies have revealed instances of nuclear-mitochondrial incompatibility by studying the progression of chimeric individuals into adulthood, MR studies in humans have been conducted in vitro and only followed embryos through the blastula stage of development. This has led to calls for additional preclinical trials of MR therapy with a much longer time horizon [9]. However, it has occurred to us that long term experiments of this kind have already been conducted in nature via the process of human evolution. The evolutionary history of anatomically modern humans has been characterized by relatively long periods of isolation and genetic divergence interspersed with migrations and genetic admixture between previously isolated populations [28-30]. Admixture between genetically distinct human populations should have brought together divergent nuclear and mitochondrial genomes. Evidence that this is in fact the case could be taken to refute the nuclear-mitochondrial genome mismatch hypothesis for humans and to thereby support the feasibility of MR therapy.

In light of this realization, we systematically evaluated the extent of naturally occurring nuclear-mitochondrial genome swapping that has occurred among human populations. The goal of our survey was to get an idea of the extent of nuclear-mitochondrial divergence that can be tolerated within any single individual as well as a sense of how often swapping has occurred in human evolution. To do this, we evaluated the distribution of nuclear genomic diversity and mtDNA haplotypes among the 26 human populations, representing five major continental groups, which were characterized via whole genome sequencing as part of the 1000 Genomes Project (1KGP) [31]. We also performed a confirmatory analysis of mtDNA sequence variation among 58 populations from the Human Genome Diversity Project (HGDP), which characterized mitochondrial genomes to a lower level of resolution using SNP arrays [29]. We reasoned that since the donors for these human genome diversity projects are (apparently) healthy individuals, they should not bear incompatible nuclearmitochondrial genome combinations. In addition, since these diverse populations have been shaped by millennia of natural selection, deleterious combinations of nuclearmitochondrial genomes should have been eliminated long ago and would not be observed in extant populations. In this sense, our survey can be considered as a test of the nuclear-mitochondrial genome incompatibility hypothesis in humans.

\section{Methods}

\section{Human population genomic data}

Human genome sequence variants, characterized via whole genome sequencing of healthy donors, for nuclear DNA (nDNA) and mitochondrial DNA (mtDNA) were obtained from the 1KGP [31] data portal [32]. The data analyzed here correspond to the Phase 3 release of 1KGP [31], with variants available for 2,054 individuals from 26 populations representing five major continental population groups (Table 1). For the purposes of this study, we consider the ASW and ACB populations to be members of the Admixed American continental population group.

HGDP mtDNA sequence variants from healthy donors characterized via SNP arrays [29] were obtained from the HGDP-CEPH Genome Diversity Panel Database (version 3.0) [33]. The HGDP data analyzed here correspond to the Dataset 2 release from September 2007, with variants available for 1,043 individuals from 58 populations representing six major continental population groups (Additional file 1: Table S1).

\section{Nuclear and mitochondrial genetic divergence}

Genetic divergence levels between pairs of individuals, for nDNA and mtDNA, were measured as allele sharing distances [34] as implemented in PLINK v1.90 [35]. Allele sharing distances are calculated as the number of different variants $(d)$ normalized by the total number of sites $(2 n)$ under consideration. The resulting nDNA and mtDNA pairwise distance matrices were projected in two dimensional space using multi-dimensional scaling (MDS) [36] implemented in $\mathrm{R}$ [37, 38]. Allele sharing distances for nDNA and mtDNA were used to reconstruct a neighbor-joining phylogenetic trees [39] using the program MEGA [40]. nDNA versus mtDNA allele sharing distances were regressed, and the resulting scatterplot was visualized using a smoothed color density representation in $\mathrm{R}$. The Spearman correlation coefficient was used to quantify the correlation between nDNA and mtDNA distances and the significance of the relationship. Genetic divergence levels for nDNA and mtDNA were ranked separately, and the ranks were 
Table 11000 Genomes Project (1KGP) populations analyzed in this study

\begin{tabular}{|c|c|c|c|c|c|c|c|}
\hline & Short & Full description & $N$ & & Short & Full description & N \\
\hline \multirow{5}{*}{$\begin{array}{l}\text { Africa } \\
(n=504)\end{array}$} & ESN & Esan in Nigeria & 99 & \multirow{5}{*}{$\begin{array}{l}\text { India } \\
(n=489)\end{array}$} & BEB & Bengali in Bangladesh & 86 \\
\hline & GWD & $\begin{array}{l}\text { Gambian in Western Division, } \\
\text { The Gambia }\end{array}$ & 113 & & $\mathrm{GIH}$ & Gujarati Indian in Houston, TX & 103 \\
\hline & LWK & Luhya in Webuye, Kenya & 99 & & ITU & Indian Telugu in the UK & 102 \\
\hline & MSL & Mende in Sierra Leone & 85 & & PJL & Punjabi in Lahore, Pakistan & 96 \\
\hline & YRI & Yoruba in Ibadan, Nigeria & 108 & & STU & Sri Lankan Tamil in the UK & 102 \\
\hline \multirow{5}{*}{$\begin{array}{l}\text { East Asia } \\
(n=504)\end{array}$} & CDX & Chinese Dai in Xishuangbanna, China & 93 & \multirow{10}{*}{$\begin{array}{l}\text { America } \\
(n=504)\end{array}$} & $A C B$ & African Caribbean in Barbados & 96 \\
\hline & $\mathrm{CHB}$ & Han Chinese in Bejing, China & 103 & & ASW & African Ancestry in Southwest US & 61 \\
\hline & $\mathrm{CHS}$ & Southern Han Chinese, China & 105 & & CLM & Colombian in Medellin, Colombia & 94 \\
\hline & JPT & Japanese in Tokyo, Japan & 104 & & MXL & $\begin{array}{l}\text { Mexican Ancestry in Los Angeles, } \\
\text { California }\end{array}$ & 64 \\
\hline & KHV & Kinh in Ho Chi Minh City, Vietnam & 99 & & PEL & Peruvian in Lima, Peru & 85 \\
\hline \multirow[t]{5}{*}{$\begin{array}{l}\text { Europe } \\
(n=503)\end{array}$} & CEU & $\begin{array}{l}\text { Utah residents with NW European } \\
\text { ancestry }\end{array}$ & 99 & & PUR & Puerto Rican in Puerto Rico & 104 \\
\hline & FIN & Finnish in Finland & 99 & & & & \\
\hline & GBR & British in England and Scotland & 91 & & & & \\
\hline & IBS & Iberian populations in Spain & 107 & & & & \\
\hline & TSI & Toscani in Italy & 107 & & & & \\
\hline
\end{tabular}

The continental population groups, short three letter symbols, full population name and number of individuals from each population are shown. The continental population groups correspond to the convention used by the $1 \mathrm{KGP}$ with the exception of the ASW and ACB populations, which we consider as part of the admixed American population group

compared in order to calculate nDNA versus mtDNA distance-differences.

\section{mtDNA haplotype analysis}

For both the 1KGP and the HGDP data, mitochondrial haplotypes were determined from mtDNA sequence variants using the HaploGrep2 program [41]. Evolutionary relationships among mtDNA haplogroups were taken from the PhyloTreemt website [42]. Counts of mtDNA haplogroups were determined for the individual populations, and the counts were hierarchically clustered according to the continental population groups from the 1KGP and HGDP along with the origins of their previously characterized macro-haplogroups. 1KGP mtDNA haplotypes were determined based on 3,892 sequence variants from whole genome sequencing, whereas HGDP mtDNA haplotypes were determined based on 162 variants from SNP arrays.

\section{Results and discussion}

\section{Comparison of nuclear versus mitochondrial genetic divergence}

The nuclear-mitochondrial mismatch hypothesis rests on the idea that nuclear and mitochondrial genomes coevolve as populations diverge and thus can be taken to predict that nuclear DNA (nDNA) and mitochondrial DNA (mtDNA) divergence levels will be correlated. In other words, closely related pairs of individuals (from within populations) should show low levels of both
nDNA and mtDNA divergence, whereas distantly related individuals (from between populations) should have relatively divergent nuclear and mitochondrial sequences. To evaluate this prediction, we computed the nDNA and mtDNA allele sharing distances between all pairs of individuals from the 1KGP as described in the Materials and Methods. The 1KGP entailed the characterization of nuclear and mitochondrial genome sequences of 2,504 individuals across a broad range of human population genetic diversity: 26 populations representing five major continental population groups (Table 1). Multidimensional scaling (MDS) was used to plot the evolutionary relationships among individuals in two dimensions (components $1 \& 2$ in Fig. 1a \& b) based on the nDNA and mtDNA distances. The genetic distances calculated for nuclear DNA accurately reflect known evolutionary relationships among human populations (Fig. 1a) $[29,30]$. African, East Asian and European populations occupy the three poles of human genetic diversity with African populations relatively distinct from the others. Admixed populations from India and the Americas occupy intermediate positions according the relative ancestry contributions from ancient source populations.

Genetic distances calculated from mtDNA sequences also reveal substantial genetic structure among human populations (Fig. 1b). In the case of mtDNA, the major groups correspond very well with previously characterized mtDNA haplogroups (Additional file 1: Figure S1) [42]. The primary MDS component separates the set of 

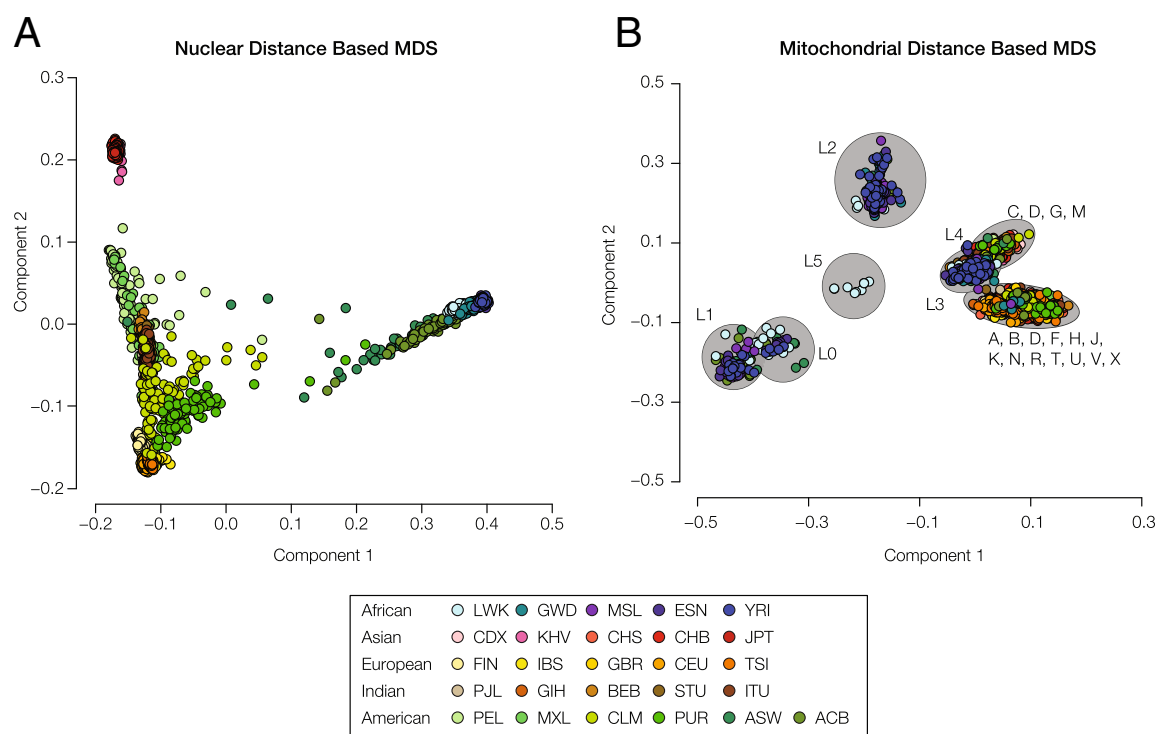

C

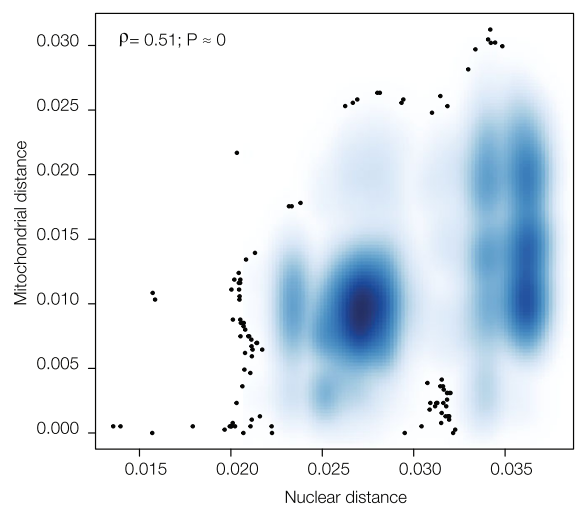

D

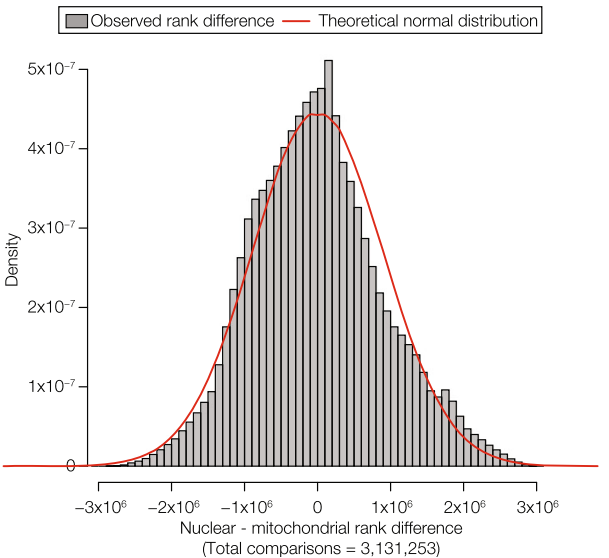

Fig. 1 Comparison of nuclear (nDNA) versus mitochondrial (mtDNA) genetic divergence levels. Genetic divergence levels between all pairs of human individuals from the 1KGP were calculated as described in the Materials and Methods. a Multidimensional scaling (MDS) plot showing the evolutionary relationships among the 1KGP individuals based on their nuclear (nDNA) genetic distances. b MDS plot showing the evolutionary relationships among the 1KGP individuals based on their mitochondrial (mtDNA) genetic distances. Mitochondrial haplogroup designations are shown on the plot and macro-haplogroups are indicated by grey circles. For panels A \& B, individuals from different populations are color coded as shown in the key. $\mathbf{c}$ Density scatterplot showing the regression of nuclear (x-axis) against mitochondrial (y-axis) genetic distances for all pairs of individuals. Denser regions of points are shown in dark blue; outlier points are indicated as black dots. The Spearman correlation coefficient ( $\rho$ ) and corresponding $P$-value are shown. $\mathbf{d}$ Distribution of the nuclear versus mitochondrial distance-differences. A theoretical normal distribution (red line) is superimposed over the observed distribution (grey bars)

ancient L mtDNA haplogroups (L0, L1 \& L5) from the more derived L haplogroups (L3 \& L4), which cluster with the rest of the derived haplogroups. The MDS clusters of the derived mtDNA haplogroups $(M, N \& R)$ also correspond well with the previously known classification; although, the mtDNA genetic distances provided relatively little resolution within these subgroups.

We regressed the nDNA versus mtDNA pairwise genetic distances to test for the correlation predicted by the nuclear-mitochondrial mismatch hypothesis. Overall, nDNA and mtDNA genetic distances are highly correlated, consistent with the prediction (Fig. 1c). Analysis of 2,504 individuals from the $1 \mathrm{KGP}$ yields $>3$ million pairwise comparisons, and the $\mathrm{nDNA}$ and $\mathrm{mtDNA}$ genetic distances are correlated at $r=0.51, P \approx 0$. Despite the high overall correlation between $\mathrm{nDNA}$ and mtDNA genetic distances, there is a substantial amount of spread in the differences observed for pairs of nDNA versus mtDNA distances (Fig. 1c). There are numerous pairs of individuals, the outliers in the distance-difference distribution (Fig. 1d), that have very closely related nuclear genomes and distantly related mitochondrial genomes or vice versa. These observations point 
to healthy (viable) individuals that nevertheless have potentially mismatched nuclear and mitochondrial genomes. We attempted to further evaluate this possibility by analyzing the distribution of mtDNA haplotypes among global human populations.

\section{Global distribution of mtDNA haplotypes}

Human mtDNA haplotypes are widely used as markers of maternal ancestry, and accordingly the continental origins of mtDNA haplotype groups are well known [43]. Analysis of nuclear DNA can also be used to resolve evolutionary relationships among human populations and for individual ancestry assignment $[29,30]$. The 1 KG data (Table 1) provide an opportunity to compare the global distributions of human mitochondrial and nuclear genetic diversity and to test the hypothesis of nuclear-mitochondrial genome incompatibility among naturally occurring populations. Mitochondrial sequence variants for the 1KGP individuals were converted into mtDNA haplotypes using the HaploGrep2 program [41] as described in the Materials and Methods. The distributions of corresponding mtDNA haplogroups were characterized for the 26 populations of the 1KGP as shown in Fig. 2a. The observed global distributions of these mtDNA haplogroups correspond well with the previously characterized origins of mtDNA haplogroups. For example, the ancestral L haplogroup predominates in Africa [44], whereas the D and F haplogroups are most frequent in East Asia [45]. The H, U and $\mathrm{T}$ haplogroups are most common in Europe [46].

The observed numbers of each mtDNA haplogroup were recorded for each individual population and clustered into the five major population groups as shown in Fig. 2b. This allowed us to evaluate the extent to which observed mtDNA haplogroups correspond to their expected continent (or broad geographic region) of origin. The African, East Asian and European populations show very coherent patterns of mtDNA haplogroup distributions, whereas the Indian and American population groups show more divergent haplogroups consistent with their admixed origins. Indian populations show a combination of largely European and Asian mtDNA haplogroups, consistent with relatively ancient human migration and admixture events that formed these populations $[47,48]$. Interestingly, the Gujarati $(\mathrm{GIH})$ population from Western India shows several instances of African haplogroups, perhaps consistent with subsequent migrations across the Indian Ocean or along the coast. The American populations from the 1KGP were formed by more recent admixture between European, Native American and African source populations [49-51]. Accordingly, individuals from these populations show mtDNA haplogroups corresponding to each of these regions. Native American mtDNA haplogroups are most common among the four admixed Latino populations
(CLM, MXL, PEL and PUR), whereas African mtDNA haplogroups are most common among the AfricanAmerican (ASW) and Afro-Caribbean (ACB) populations. The prevalence of Native American haplotypes in Latino populations is not necessarily correlated with their inferred ancestry based on nuclear DNA. For example, 67\% of mtDNA haplotypes from Puerto Rico have a Native American origin, and 13\% have a European origin; analysis of nuclear DNA, on the other hand, indicates that the same population has $72 \%$ European ancestry compared to only $13 \%$ Native American ancestry.

While the co-occurrence of nuclear and mitochondrial genomes with distinct ancestries in admixed populations may be expected, it is nevertheless inconsistent with the nuclear-mitochondrial genome incompatibility hypothesis. Perhaps even more strikingly, there are a number of mismatched nuclear-mitochondrial genome pairs among presumably non-admixed populations. For example, individuals from African populations in Gambia (GWD) and Sierra Leone (MSL) have the U mtDNA haplogroup that is most often found in Europe and India. The specific $U$ mtDNA haplotypes found in these populations all correspond to the U6 haplogroup. This haplogroup has a Near East origin followed by expansion into North Africa [52]. The presence of this haplogroup in West African populations likely reflects subsequent contact with North African groups [53]. A single individual from the Beijing population ( $\mathrm{CHB}$ ) of the East Asian continental group was found to have a $\mathrm{K}$ mtDNA haplogroup, which is not known to be found in East Asia [54]. Several individuals from European populations in Spain (IBS) and Italy (TSI) have African L mtDNA haplogroups. This likely reflects relatively ancient African admixture that has been documented for Southern European populations [55]. These same two European populations also have individuals with more typically Asian mtDNA haplotypes from the D, N and R haplogroups. It should be noted that these particular haplogroups are widespread and have previously been found in Europe [45]. This result however underscores the point that members of the same haplogroup can co-occur with nuclear genomes that have very distinct genetic ancestries.

We performed a replication analysis of the global distribution of mtDNA haplotypes using mtDNA sequence variants characterized with SNP arrays as part of the HGDP. While the SNP array data from this project provide substantially less resolution than the sequence data from the 1KGP 162 mtDNA variants for HGDP compared to 3,892 variants for $1 \mathrm{KGP}$ - we were still able to infer mtDNA haplotypes from the HGDP variant data, albeit at a more granular level. Nevertheless, the HGDP data also show a number of cases of nuclear-mitochondrial lineage mismatches, thereby contradicting the nuclear-mitochondrial mismatch hypothesis (Additional file 2: Table S2). 


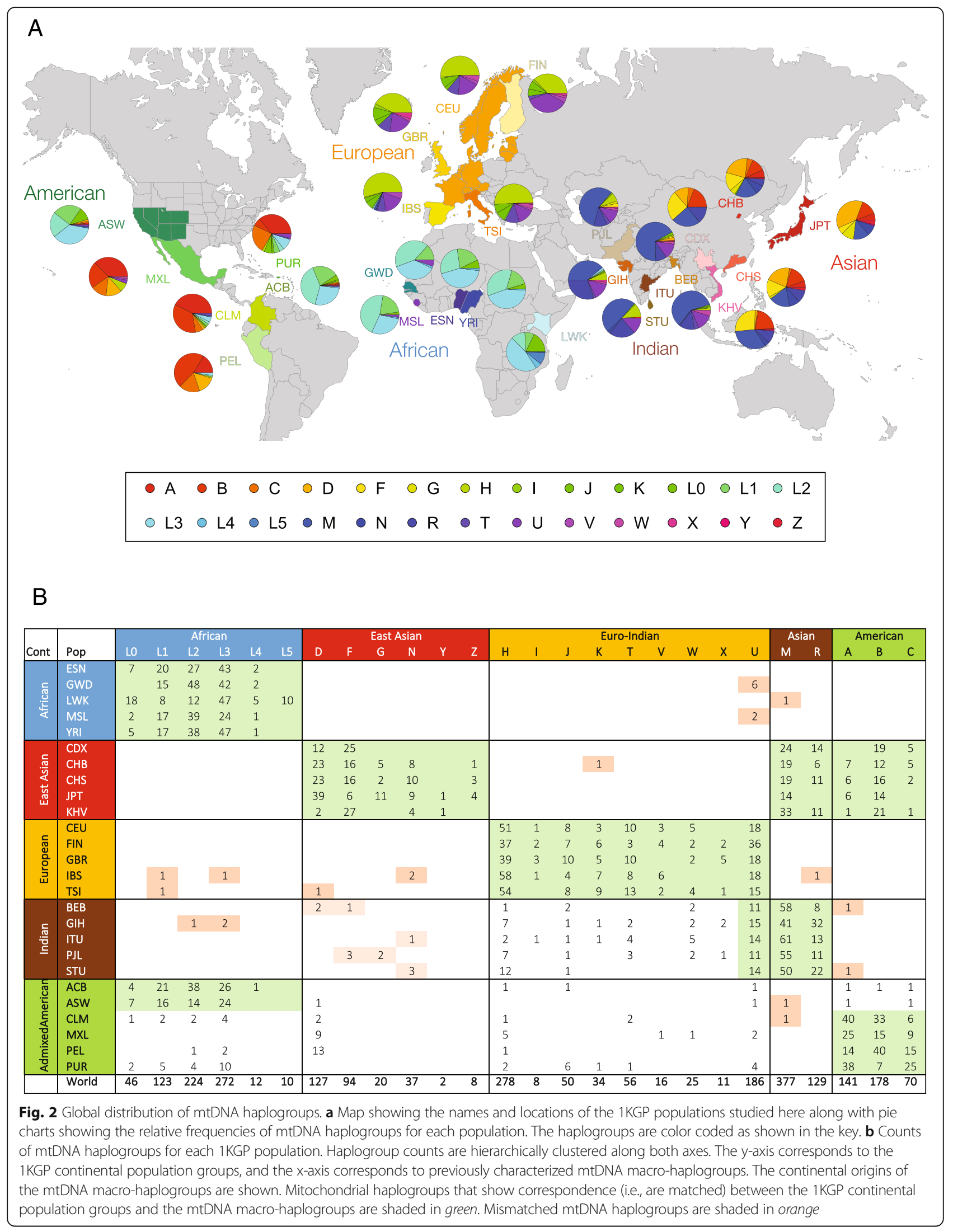


As an additional control analysis, we performed a similar comparison of the distribution of the Y-DNA haplotypes across the populations of the 1KGP. The cooccurrence of Y-DNA and nuclear genomes with distinct ancestries can also be observed for this dataset; however, this appears to occur less often than seen for mtDNA (Additional file 3: Table S3). The slight difference between the mtDNA and Y-DNA results is consistent with previous work showing sex-specific patterns of human migration characterized by relatively lower levels of male migration, and higher levels of female migration, based on the phenomenon of patrilocality [56].

\section{Phylogenetic discordance of mtDNA haplotypes}

Given the existence of a number of population mismatched mtDNA haplotypes, as described in the previous section, we used a phylogenetic approach to more directly compare nDNA genetic distances to the distribution of mtDNA haplotypes. The nDNA genetic distances were used to compute a phylogenetic tree relating all individuals from the $1 \mathrm{KGP}$, and individuals' mtDNA haplotypes were then considered in the context of this tree (Fig. 3). Populations belonging to the five major continental groups are clearly resolved along this phylogeny, underscoring the extent to which nuclear genetic divergence recapitulates human evolutionary history. The only exception is the placement of the admixed American populations according to their relative ancestry proportions. The African-American populations ASW and ACB group together with the other African populations, whereas the Peruvian population (PEL) occupies an intermediate position owing to its relatively high levels of




Native American ancestry. These same patterns can be observed in the nDNA distance MDS plot (Fig. 1a).

The phylogenetic placement of the mtDNA haplotypes also highlights the extent of naturally occurring nuclearmitochondrial genome mismatch that can be seen for human populations. Pairs of individuals with very low nDNA divergence levels, i.e. sister taxa on the nDNA tree shown in Fig. 3, can have mtDNA haplotypes that are extremely divergent (see mtDNA haplogroup tree in Additional file 1: Figure S1). For example, two European individuals with L1 haplotypes, which correspond to one of the most ancient African mtDNA haplogroups, are most closely related to individuals with the highly derived $\mathrm{H}$ mtDNA haplogroup. Similarly, an Indian individual with an ancient African L2 mtDNA haplotype is most closely related to an individual with the highly derived U mtDNA haplotype. In Africa, U mtDNA haplotypes are paired with more ancient $\mathrm{L}$ haplotypes reflecting gene flow from the Near East back into Africa as previously discussed.

\section{Conclusion}

The results of our analysis on naturally occurring human genetic variation show that nuclear and mitochondrial genomes from divergent human populations can coexist within presumably healthy individuals, indicating that such mismatched nDNA-mtDNA combinations are not deleterious and have not been eliminated by purifying selection. In other words, the long and ongoing experiment of human evolution provides no evidence whatsoever in support of the nuclear-mitochondrial mismatch hypothesis. These results can be taken to support the feasibility, and potential safety, of MR-assisted in vitro fertilization, at least with respect to the compatibility of human nDNA and mtDNA genomes. Of course, our results do not bear on any potential complications related to the technical implementation of such a complicated procedure. For example, it is extremely difficult to ensure that none of the defective mitochondria are transferred along with the nuclear genome. Indeed, it was recently shown that even when only a small percentage of defective mitochondria are carried over in the nuclear transfer process, they can increase in copy number and eventually replace most or all of the healthy mitochondria from the egg donor [57]. Such technical hurdles will need to be addressed to ensure the maximum safety of MR therapy.

Our results are in conflict with a number of previous studies on model organisms, which provide numerous lines of evidence in support of nuclear-mitochondrial genome incompatibility. For example, studies in mice have shown physical and neurological deficits related to nuclear-mitochondrial mismatches $[14,15]$. In addition, the co-occurrence of divergent nuclear and mitochondrial genomes in invertebrates has been associated with diminished mitochondrial function $[25,26]$ along with deleterious effects on aging [16-19], survival [20] and fertility [21-24]. When considered together, these previous studies have been taken to issue a strong note of caution against MR therapy $[9,11]$.

It is interesting to note that much of the resistance to MR therapy has been articulated by evolutionary biologists who emphasize the extent to which nuclear and mitochondrial genomes co-evolve along population lineages [11]. This realization has raised the seemingly legitimate concern that advocates of MR therapy, and/or the regulatory bodies that are charged with evaluating its safety, may not have adequately considered the implications of evolution for the implementation of this new technology. However, the results of our study suggest that the model organism studies that have been used to argue against the safety of MR therapy do not accurately reflect the nature of human evolution [27]. For the most part, these model organism studies relied on backcrossing and the generation of inbred lines, and they also involved relatively divergent populations. Experiments of this kind can be expected to result in extremes of nuclear-mitochondrial genome divergence. Human populations, on the other hand, tend to show both low levels of genetic divergence and low inbreeding. Accordingly, one may expect to see less pronounced effects of nuclearmitochondrial mismatch in human populations, and that is exactly what we observed in our study.

\section{Additional files}

Additional file 1: Table S1. HGDP populations analyzed in this study. Figure S1. (A) Phylogenetic tree based on mtDNA haplotype genetic distances and (B) dendogram showing previously defined relationships among major mtDNA haplogroups. (DOCX $344 \mathrm{~kb}$ )

Additional file 2: Table S2. Counts of mtDNA haplogroups for the HGDP populations analyzed here. Global population distributions of mtDNA haplogroups are organized as shown for the 1KGP in Table 1. (XLSX $14 \mathrm{~kb}$ )

Additional file 3: Table S3. Counts of Y-DNA haplogroups for the 1KGP populations analyzed here. Global population distributions of Y-DNA haplogroups are organized as shown for the mtDNA haplogroups in Table 1. (XLSX $13 \mathrm{~kb}$ )

\section{Abbreviations}

1KGP: 1000 genomes project; HFEA: Human fertilisation and embryology authority; IVF: in vitro fertilization; MDS: Multidimensional scaling; MR: Mitochondrial replacement; mtDNA: mitochondrial DNA; nDNA: nucleosomal DNA

\section{Acknowledgement}

The authors thank Emily T. Norris for feedback on the manuscript.

\section{Funding}

LR and IKJ were supported by Georgia Institute of Technology Bioinformatics Graduate Program and the IHRC-GIT Applied Bioinformatics Laboratory (ABiL). The funding body played no role in the collection, analysis, and interpretation of data or in writing the manuscript. 


\section{Availability of data and materials}

All data supporting our findings can be accessed via the 1000 Genomes Project website http://www.internationalgenome.org/ and the Human Genome Diversity Project website http://www.hagsc.org/hgdp/

\section{Authors' contributions}

IKJ and LR conceived of and designed the study. LR performed all the analyses. IKJ and LR wrote the final manuscript. Both authors read and approved the final manuscript.

\section{Authors' information}

$L R$ is a PhD student in the Bioinformatics graduate program at Georgia Tech and team lead of the Applied Bioinformatics Laboratory (ABiL). His research interests include computationally-enabled human population and clinical genomic analyses.

IKJ is Associate Professor in the School of Biology and Director of the Bioinformatics graduate program at Georgia Tech. He is the co-founder of the PanAmerican Bioinformatics Institute. His research interests include computational genomic approaches to human evolution and health.

\section{Competing interests}

The authors declare that they have no competing interests.

\section{Consent for publication}

Not applicable.

\section{Ethics approval and consent to participate}

This study uses publicly available, unrestricted, de-identified human genome sequence variant data from the 1000 Genomes Project and the Human Genome Diversity Project and therefore does not require ethics committee approval.

\section{Author details}

${ }^{1}$ School of Biology, Georgia Institute of Technology, Atlanta, GA, USA. ${ }^{2}$ PanAmerican Bioinformatics Institute, Cali, Colombia. ${ }^{3}$ Applied Bioinformatics Laboratory, Atlanta, GA, USA

Received: 27 September 2016 Accepted: 2 February 2017 Published online: 08 February 2017

\section{References}

1. Schon EA, DiMauro S, Hirano M. Human mitochondrial DNA: roles of inherited and somatic mutations. Nat Rev Genet. 2012:13(12):878-90.

2. Taylor RW, Turnbull DM. Mitochondrial DNA mutations in human disease. Nat Rev Genet. 2005;6(5):389-402.

3. Wallace DC, Chalkia D. Mitochondrial DNA genetics and the heteroplasmy conundrum in evolution and disease. Cold Spring Harb Perspect Biol. 2013; 5(11):a021220

4. Sato A, Kono T, Nakada K, Ishikawa K, Inoue S, Yonekawa H, Hayashi J. Gene therapy for progeny of mito-mice carrying pathogenic mtDNA by nuclear transplantation. Proc Natl Acad Sci U S A. 2005;102(46):16765-70.

5. Tachibana M, Sparman M, Sritanaudomchai H, Ma H, Clepper L, Woodward J, Li Y, Ramsey C, Kolotushkina O, Mitalipov S. Mitochondrial gene replacement in primate offspring and embryonic stem cells. Nature. 2009; 461(7262):367-72.

6. Tachibana M, Amato P, Sparman M, Woodward J, Sanchis DM, Ma H, Gutierrez NM, Tippner-Hedges R, Kang E, Lee HS, et al. Towards germline gene therapy of inherited mitochondrial diseases. Nature. 2013:493(7434):627-31.

7. Craven L, Tuppen HA, Greggains GD, Harbottle SJ, Murphy JL, Cree LM, Murdoch AP, Chinnery PF, Taylor RW, Lightowlers RN, et al. Pronuclear transfer in human embryos to prevent transmission of mitochondrial DNA disease. Nature. 2010;465(7294):82-5.

8. Hyslop LA, Blakeley P, Craven L, Richardson J, Fogarty NM, Fragouli E, Lamb M, Wamaitha SE, Prathalingam N, Zhang Q, et al. Towards clinical application of pronuclear transfer to prevent mitochondrial DNA disease. Nature. 2016; 534(7607):383-6.

9. Reinhardt K, Dowling DK, Morrow EH. Medicine. Mitochondrial replacement, evolution, and the clinic. Science. 2013;341(6152):1345-6.

10. First 'three person baby' born using new method. http://www.bbc.com/ news/health-37485263. Accessed 27 Sept 2016.
11. Hamilton G. The hidden risks for 'three-person' babies. Nature. 2015; 525(7570):444-6.

12. da Fonseca RR, Johnson WE, O'Brien SJ, Ramos MJ, Antunes A. The adaptive evolution of the mammalian mitochondrial genome. BMC Genomics. 2008;9:119.

13. Osada N, Akashi H. Mitochondrial-nuclear interactions and accelerated compensatory evolution: evidence from the primate cytochrome $\mathrm{C}$ oxidase complex. Mol Biol Evol. 2012;29(1):337-46.

14. Nagao $\mathrm{Y}$, Totsuka $\mathrm{Y}$, Atomi $\mathrm{Y}$, Kaneda $\mathrm{H}$, Lindahl KF, Imai H, Yonekawa $\mathrm{H}$. Decreased physical performance of congenic mice with mismatch between the nuclear and the mitochondrial genome. Genes Genet Syst. 1998;73(1):21-7.

15. Roubertoux PL, Sluyter F, Carlier M, Marcet B, Maarouf-Veray F, Cherif C, Marican C, Arrechi P, Godin F, Jamon M, et al. Mitochondrial DNA modifies cognition in interaction with the nuclear genome and age in mice. Nat Genet. 2003;35(1):65-9.

16. Camus MF, Clancy DJ, Dowling DK. Mitochondria, maternal inheritance, and male aging. Curr Biol. 2012;22(18):1717-21.

17. Dowling DK, Maklakov AA, Friberg U, Hailer F. Applying the genetic theories of ageing to the cytoplasm: cytoplasmic genetic covariation for fitness and lifespan. J Evol Biol. 2009:22(4):818-27.

18. Clancy DJ. Variation in mitochondrial genotype has substantial lifespan effects which may be modulated by nuclear background. Aging Cell. 2008; $7(6): 795-804$

19. Maklakov AA, Friberg U, Dowling DK, Arnqvist G. Within-population variation in cytoplasmic genes affects female life span and aging in Drosophila melanogaster. Evolution. 2006;60(10):2081-6.

20. Dowling DK, Meerupati T, Arnqvist G. Cytonuclear interactions and the economics of mating in seed beetles. Am Nat. 2010;176(2):131-40.

21. Yee $\mathrm{WK}$, Sutton $\mathrm{KL}$, Dowling $\mathrm{DK}$. In vivo male fertility is affected by naturally occurring mitochondrial haplotypes. Curr Biol. 2013;23(2):R55-6.

22. Innocenti P, Morrow EH, Dowling DK. Experimental evidence supports a sex-specific selective sieve in mitochondrial genome evolution. Science. 2011;332(6031):845-8.

23. Clancy DJ, Hime GR, Shirras AD. Cytoplasmic male sterility in Drosophila melanogaster associated with a mitochondrial CYTB variant. Heredity. 2011;107(4):374-6.

24. Dowling DK, Nowostawski AL, Arnqvist G. Effects of cytoplasmic genes on sperm viability and sperm morphology in a seed beetle: implications for sperm competition theory? J Evol Biol. 2007;20(1):358-68.

25. Burton RS, Ellison CK, Harrison JS. The sorry state of F2 hybrids: consequences of rapid mitochondrial DNA evolution in allopatric populations. Am Nat. 2006;168 Suppl 6:S14-24

26. Sackton TB, Haney RA, Rand DM. Cytonuclear coadaptation in Drosophila: disruption of cytochrome c oxidase activity in backcross genotypes. Evolution. 2003;57(10):2315-25.

27. Chinnery PF, Craven L, Mitalipov S, Stewart JB, Herbert M, Turnbull DM. The challenges of mitochondrial replacement. PLoS Genet. 2014;10(4):e1004315.

28. Hellenthal G, Busby GB, Band G, Wilson JF, Capelli C, Falush D, Myers S. A genetic atlas of human admixture history. Science. 2014;343(6172):747-51.

29. Li JZ, Absher DM, Tang H, Southwick AM, Casto AM, Ramachandran S, Cann HM, Barsh GS, Feldman M, Cavalli-Sforza LL, et al. Worldwide human relationships inferred from genome-wide patterns of variation. Science. 2008;319(5866):1100-4.

30. Rosenberg NA, Pritchard JK, Weber JL, Cann HM, Kidd KK, Zhivotovsky LA, Feldman MW. Genetic structure of human populations. Science. 2002 298(5602):2381-5

31. Genomes Project C, Auton A, Brooks LD, Durbin RM, Garrison EP, Kang HM, Korbel JO, Marchini JL, McCarthy S, McVean GA, et al. A global reference for human genetic variation. Nature. 2015;526(7571):68-74.

32. A global reference for human genetic variation. ftp://ftp.1000genomes.ebi. ac.uk/vol1/ftp/release/20130502/. Accessed 1 Oct 2015.

33. HGDP-CEPH Genome Diversity Panel Database. http://www.cephb.fr/hgdp/. Accessed 15 Dec 2016.

34. Gao X, Martin ER. Using allele sharing distance for detecting human population stratification. Hum Hered. 2009;68(3):182-91.

35. Purcell $S$, Neale B, Todd-Brown $K$, Thomas L, Ferreira MA, Bender D, Maller J, Sklar P, de Bakker PI, Daly MJ, et al. PLINK: a tool set for whole-genome association and population-based linkage analyses. Am J Hum Genet. 2007; 81(3):559-75.

36. Gower JC. Some distance properties of latent root and vector methods used in multivariate analysis. Biometrika. 1966;53(3-4):325-38.

37. Ihaka R, Gentleman R. R: a language for data analysis and graphics. J Comput Graph Stat. 1996;5(3):299-314. 
38. Mardia KV, Kent JT, Bibby JM. Multivariate analysis. 1980.

39. Saitou N, Nei M. The neighbor-joining method: a new method for reconstructing phylogenetic trees. Mol Biol Evol. 1987;4(4):406-25.

40. Kumar S, Stecher G, Tamura K. MEGA7: Molecular Evolutionary Genetics Analysis Version 7.0 for Bigger Datasets. Mol Biol Evol. 2016;33(7):1870-4.

41. Weissensteiner $H$, Pacher D, Kloss-Brandstatter A, Forer L, Specht G, Bandelt HJ, Kronenberg F, Salas A, Schonherr S. HaploGrep 2: mitochondrial haplogroup classification in the era of high-throughput sequencing. Nucleic Acids Res. 2016:44(W1):W58-63.

42. van Oven M, Kayser M. Updated comprehensive phylogenetic tree of global human mitochondrial DNA variation. Hum Mutat. 2009;30(2):E386-94.

43. Cann RL, Stoneking M, Wilson AC. Mitochondrial DNA and human evolution. Nature. 1987;325(6099):31-6.

44. Chen YS, Torroni A, Excoffier L, Santachiara-Benerecetti AS, Wallace DC. Analysis of mtDNA variation in African populations reveals the most ancient of all human continent-specific haplogroups. Am J Hum Genet. 1995;57(1): 133-49.

45. Comas D, Plaza S, Wells RS, Yuldaseva N, Lao O, Calafell F, Bertranpetit J. Admixture, migrations, and dispersals in Central Asia: evidence from maternal DNA lineages. Eur J Hum Genet. 2004;12(6):495-504.

46. Torroni A, Huoponen K, Francalacci P, Petrozzi M, Morelli L, Scozzari R, Obinu D, Savontaus ML, Wallace DC. Classification of European mtDNAs from an analysis of three European populations. Genetics. 1996;144(4):1835-50.

47. Moorjani P, Thangaraj K, Patterson N, Lipson M, Loh PR, Govindaraj P, Berger B, Reich D, Singh L. Genetic evidence for recent population mixture in India. Am J Hum Genet. 2013;93(3):422-38.

48. Kivisild T, Bamshad MJ, Kaldma K, Metspalu M, Metspalu E, Reidla M, Laos S, Parik J, Watkins WS, Dixon ME, et al. Deep common ancestry of indian and western-Eurasian mitochondrial DNA lineages. Curr Biol. 1999;9(22):1331-4.

49. Rishishwar L, Conley AB, Wigington $\mathrm{CH}$, Wang L, Valderrama-Aguirre $\mathrm{A}$, Jordan IK. Ancestry, admixture and fitness in Colombian genomes. Sci Rep. 2015;5:12376.

50. Wang S, Ray N, Rojas W, Parra MV, Bedoya G, Gallo C, Poletti G, Mazzotti G, Hill K, Hurtado AM, et al. Geographic patterns of genome admixture in Latin American Mestizos. PLoS Genet. 2008;4(3):e1000037.

51. Bryc K, Velez C, Karafet T, Moreno-Estrada A, Reynolds A, Auton A, Hammer M, Bustamante CD, Ostrer H. Colloquium paper: genome-wide patterns of population structure and admixture among Hispanic/Latino populations. Proc Natl Acad Sci U S A. 2010:107 Suppl 2:8954-61.

52. Maca-Meyer N, Gonzalez AM, Pestano J, Flores C, Larruga JM, Cabrera VM. Mitochondrial DNA transit between West Asia and North Africa inferred from U6 phylogeography. BMC Genet. 2003;4:15.

53. Secher B, Fregel R, Larruga JM, Cabrera VM, Endicott P, Pestano JJ, Gonzalez AM. The history of the North African mitochondrial DNA haplogroup U6 gene flow into the African, Eurasian and American continents. BMC Evol Biol. 2014;14:109.

54. Jones ER, Gonzalez-Fortes G, Connell S, Siska V, Eriksson A, Martiniano R, McLaughlin RL, Gallego Llorente M, Cassidy LM, Gamba C, et al. Upper Palaeolithic genomes reveal deep roots of modern Eurasians. Nat Commun. 2015;6:8912.

55. Moorjani P, Patterson N, Hirschhorn JN, Keinan A, Hao L, Atzmon G, Burns E, Ostrer $\mathrm{H}$, Price $A \mathrm{~L}$, Reich D. The history of African gene flow into Southern Europeans, Levantines, and Jews. PLoS Genet. 2011;7(4):e1001373.

56. Seielstad MT, Minch E, Cavalli-Sforza LL. Genetic evidence for a higher female migration rate in humans. Nat Genet. 1998;20(3):278-80.

57. Kang E, Wu J, Gutierrez NM, Koski A, Tippner-Hedges R, Agaronyan K, Platero-Luengo A, Martinez-Redondo P, Ma H, Lee Y, et al. Mitochondria replacement in human oocytes carrying pathogenic mitochondrial DNA mutations. Nature. 2016:540(7632):270-5.

\section{Submit your next manuscript to BioMed Central and we will help you at every step:}

- We accept pre-submission inquiries

- Our selector tool helps you to find the most relevant journal

- We provide round the clock customer support

- Convenient online submission

- Thorough peer review

- Inclusion in PubMed and all major indexing services

- Maximum visibility for your research

Submit your manuscript at www.biomedcentral.com/submit 